\title{
La escuela: un espacio de promocion de salud
}

\author{
Albertina Mitjáns Martínez \\ Universidad de ia Habana \\ Universidade de Brasília
}

\section{School: one space to health promotion}

\begin{abstract}
Summary
There are one new health concept and that conception has implications concerning the role of school in the health promotion. There are tive principal directions to the action of the school: cultural formation, work preparation, health information presents in curriculum in general way and promotion of health by special disciplines (Physical Education, Art Education). The school has one relevant paper in relation to the personality education and as consequence in the life style of the persons in order to have health.
\end{abstract}

Key words: school and health; health promotion, health education

\section{Escola: um espaço para promoção da saúde}

\section{Resumo}

Há um novo conceito de saúde e esta concepção tem implicações concernentes ao papel de escola na promoção da saúde. Há cinco direções principais para a ação da escola: formação cultural, preparo para o trabalho, informações sobre saúde presentes no currículo de um modo geral e promoção da saúde por meio de disciplinas especiais (Educação Física, Educação Artística). A escola tem um papel relevante em relação à educação da personalidade e como consequiência no estilo de vida das pessoas para que tenham saúde.

Palavras-chave: escola e saúde, promoção da saúde, educação para saúde.

Dentro de la heterogeneidad de concepciones, teorías y prácticas que caracterizan a la Medicina y a la Psicología, puede apreciarse la emergencia de la tendencia a reconocer, cada vez más, la importancia de lo psicológico en la salud humana.

$\mathrm{Si}$ bien el problema de la enfermedad mental continúa siendo uno de los campos fundamentales de producción teórica y sobre todo, del trabajo profesional de muchos psicólogos; la producción científica y el trabajo práctico en relación a la prevención y a la promoción de salud van siendo cada vez mas numerosos.

La propia emergencia de un campo de.trabajo específico, la Psicología de la Salud (diferente a Ia tradicional Psicología Clínica), expresó esa tendencia, a pesar de las diferencias de enfoques, conceptos y objetivos con que se ha abordado.

Del énfasis en los problemas de la enfermedad y la salud mental se ha ido transitando hacia un nuevo momento, en el cual, el concepto de salud se ha tomando no sólo mas abrangente, sino, sobre todo, esencialmente diferente.

La salud comienza a definirse no por ausencia de enfermedad o por ausencia de síntomas, sino por características distintivas específicas, relacionadas, no sólo con la condición biológica, sino con la condición subjetiva y sociohistoricamente determinada del hombre.

En el marco de esa concepción, asumimos el concepto de salud elaborado por Gonzáles (1993 :3) quién expresa:

"La salud, sin embargo, és un proceso cualitativo que define el funcionamiento integral del 
organismo, integrandose de fonna sistémica lo somático y lo psíquico, formando una unidad donde la afectación de uno actúa necesariamente sobre el otro".

Y mas adelante: (p.11)

"La salud no es ausencia de sintomas sino un funcionamiento integral que aumenta y optimiza los recursos del organismo para disminuir su vulneravilidad a los diferentes agentes y procesos causantes de enfermedad".

La salud, concebida así, está en función, entre otros factores, del desarrollo de un conjunto de recursos subjetivos en el sujeto que le permita una interrelación adecuada con la situación social en que vive y con las contradiciones y conflictos que cotidianamente enfrenta.

También la salud está en función de un modo de vida sano, que en su carácter complejamente deteminado, depende, en buena medida, de los recursos subjetivos del sujeto quien es quien en definitiva lo asume.

Consideramos necesario destacar que el concepto sociológico de modo de vida, tal como está siendo trabajado por un conjunto de autores, incluye, no sólo, el conjunto de actividades vitales concretas que los sujetos realizam en condiciones socio-culturales determinadas, sino también un conjunto de factores subjetivos que expresan el carácter activo deI sujeto en su condición de sujeto de su modo de vida (Poltrony, 1989, González, 1993).

Enfatizando la importancia que para la salud, tienen las actividades que integran el modo de vida y su sentido psicológico González (1993:23) afirma:

"Sin embargo, hay un amplio espectro de enfermedades donde el modo de vida del sujeto, así. como su capacidad para enfrentar la vida sin convertirse en objeto de situaciones permanentes de estrés, son decisivos para su prevención y control, entre las cuales tenemos las enfermedades cardiovasculares, angiológicas, la diabetis, el cáncer y muchas otras, cuya manifestación, sobre todo las cardiovasculares, van a la cabeza del cuadro epidemiológico que el país presenta actualmente".

Modo de vida, salud, y personalidad están intimamente relacionados entre sí y simultaneamente están intimamente vinculados a Ia educación. Sin embargo, Ia concepción tradicional de salud, en su sentido estrecho y biologista, ha favorecido que salud y educación hayan sido concebidas, durante mucho tiempo, como dos esferas relativamente independientes.

Si bien, el surgimiento de la Educación para la Salud como un área específica de conocimiento y trabajo profesional, constituyó un importante punto de encuentro entre educación y salud, en lineas generales no se llegaron a trascender las concepciones tradicionales de salud y la Educación para la Salud ha sido enfocada, con muy pocas excepciones, sólo como la ensenanza de un conjunto de conocimientos y hábitos para prevenir la enfermedad. (González y Mitjáns, 1989).

La nueva concepción de salud que presentamos guarda una relación mas evidente con el proceso educativo. Apartir de ella podemos analizar el papel de la escuela, como una de las instituciones educativas básicas de la sociedad, en el proceso de salud.

Debemos destacar, que la promoción y la educación para la salud constituyen una función de la sociedad en su conjunto y de todas y cada una de sus instituciones. Las políticas sociales, el empleo, la cultura, los valores etc, tienen un importante y diferenciado impacto en la salud de los 
distintos grupos, capas y clases de la sociedad. La familia, los medi os de difusión masiva y la escuela constituyen importantes contextos para la educación y la promoción de la salud siempre en íntima relación con el macro contexto social en que están insertos.

Consideramos que las contribuciones de la escuela en relación a la salud humana son esenciales y múltiples. Vamos a intentar conceptualizarlas, al menos, de forma didáctica, en cinco principales que guardan entre sí estrechas interrelaciones.

Una de ellas, quizás la mas elemental, es proporcionar al sujeto una formación cultural mínima que le permita comprender los diversos mensajes sobre salud que se divulgan por diferentes vias: radio, televisión, carteles, periódicos, revistas, libros, etc.

Si bien, como veremos mas adelante, una formación letrada no garantiza linealmente que el sujeto sea capaz de asumir un modo de vida saludable, indiscutiblemente constituye una contribución muy importante en el sentido de dar la posiblidad de acceso a muchas informaciones y conocimientos relacionados con la salud, que en la cultura de la época en que vivimos se expresan, fundamentalmente, a través de formas linguísticas dominantes (lenguaje escrito, audio-visual, etc.)

Una segunda contribución es la que se relaciona con la preparación para el trabajo. La institución escolar proporciona una formación básica que constituye, dentro de un complejo conjunto de factores, un factor importante para una inserción favorable en la vida laboral, no sólo como forma de realización humana, sino también como vía de satisfacción de necessidades básicas, elementos esenciales para la salud.

Es importante destacar en relación con este aspecto, la compleja interralación escuelasociedad y especialmente la necessidad de que la sociedad garantice, con estrategias económicas y sociales adecuadas, el acceso real de todos a un proceso educativo institucionalizado de calidad. Esta cuestión resulta importante porque muchas veces la escuela (en le caso de que el nino tenga acceso a ella), se convierte, de hecho, en un espacio de exclusión, lo que se expresa en los altos índices de deserción escolar que caracterizan a muchos sistemas educativos. El fracaso es atribuido unilateralmente al alumno, quien, al no poder cumplir las exigencias de la institución, la abandona, comenzando, en la mayoría de los casos, un recorrido de frustraciones y fracasos indiscutiblemente nocivo para la salud individual y social.

Un aspecto específico en relación a la preparación para el trabajo es el referido al papel de la escuela en la orientación profesional de ninos y jóvenes. Entendemos la orientación profesional como el conjunto de acciones educativas sistémicas dirigidas, fundamentalmente, al desarrollo, en el sujeto, del conjunto de recursos psicológicos, que mediatizan la elección autodeterminada de la profesión y el vínculo positivo posterior con ella.

En ellibro La Personalidad: su educación y desarrollo (1989) analizamos en detalle el papel de la escuela y en especial, del maestro, en las diferentes etapas por las que, a nuestro juicio, atraviesa la formación profesional de la personalidad. El proceso de orientación profesional, tal como lo entendemos, es un proceso complejo cuyo centro no es la transmisión de información sobre el conjunto de alternativas profesionales, sino precisamente, el desarrollo de recursos psicológicos que le permitan al sujeto implicarse, explorar, reflexionar, problematizar y seleccionar un camino que, de hecho, como algunos autores también esbozan (Bohoslavsky, 1987, por ejemplo), se relaciona mas con la pregunta quién ser? que con la pregunta qué hacer?

La autodeterminación, como capacidad del sujeto para determinar sus acciones por sus propias reflexiones y convicciones, lejos de cualquier determinismo externo, es un importante elemento de salud psicológica. El no encontrarse a sí mismo, el funcionar por criterios externos no asumidos realmente, puede constituirse, de hecho, en un problema de salud con muy diversas 
formas de manifestación. De ahí la importancia de la educación de la capacidad de autodeterminación en general y especificamente de su expresión en la elección profesional.

Por otra parte, el ámbito laboral constituye uno de los espacios vitales de la vida adulta y en esa condición, uno de los espacios mas importantes de producción o no de salud. La actividad laboral puede estar asociada a vivencias emocionales positivas, realización personal, creatividad, etc. o también a estrés, ansiedad, o insatisfacción, por ejemplo. La inserción saludable en la vida laboral, depende de complejos factores dentro de los cuales se destacan un conjunto de recursos subjetivos que la escuela debe intencionalmente, contribuir a desarrollar: capacidades, motivaciones, seguridad, autodeterminación, flexibilidad, atutovaloración adecuada y otros.

Así las acciones sistémicas que desde la escuela se estructuren en relación con la orientación profesional y la educación para el trabajo, en su sentido mas general, amplía las posibilidades para que la institución escolar se constituya en un espacio de promoción de salud laboral.

Una tercera vía de acción de la escuela, y quizás la mas trabajada, se expresa en los conocimientos epecíficos que aporta en relación a la salud a través de muchas disciplinas del curriculum. Informaciones sobre el funcionamiento del organismo humano, sobre vías de transmisión de enfermedades y formación de hábitos higiénicos, sobre cultura alimentar y otras, resultan útiles en el proceso de educación de la salud.

Sin embargo, estos conocimientos, como otros cualesquiera, tienen que adquirir sentido para que se integren y participen activamente del sistema de regulación del comportamiento del sujeto. Si no se logra que, en el alumno, adquieram un sentido individualizado, no los incoorporará de forma efectiva a su reflexión y a su acción. Resulta extraordinariamente importante tener en cuenta este hecho al planear cualquier tipo de acción educativa en la institución escolar. La forma en que se trabajen estos conocimientos, al igual que el resto de los conocimientos que la escuela pretende enseñar, tiene que ser tal que se vinculen con las necesidades y motivaciones propias del alumno, provocando vivencias emocionales específicas, elementos esenciales para la constitución de estructuras de sentido de relativa estabilidad en la personalidad. El tipo de comunicación maestroalumno, el modelo que el propio maestro puede significar, y la diversidad de activities motivadoras y creativas que puede diseñar, constituyen elementos esenciales para ese empeño.

Una cuarta vía de participación de la escuela en la promoción de la salud la constituyen las disciplinas específicas que puedem contribuir a la construcción de un modo de vida sano. Ejemplo de ello son las disciplinas Educación Física y Educación Artística.

Cada vez se obtienen mayores evidencias científicas de la importancia del ejercicio físico para la salud. La disciplina Educación Física puede jugar un importante papel en el desarrollo de la motivación de los alumnos hacia el deporte y la actividad fisica en general así como en el desarrollo de habilidades específicas para su ejercicio. Además, la práctica de ejercicios físicos puede constituir una de las formas mas sanas de utilización del tiempo libre y adquire entonces un dobre valor.

Sin embargo, no puede pensarse, que por el mero hecho de formar parte del curriculum, la disciplina Educación Física va a jugar el papel al que nos referimos. Como vimos anteriormente, esto dependerá de cómo se vincule con la estructura de sentidos del sujeto en lo cual, la acción del profesor vuelve a resultar esencial.

En ocasiones el caráter estandarizado, formal y mecánico con que se imparte la Educación Física, genera lo contrario a lo que se pretende desarrollar: muchos niños comienzan a rechazar las actividades físicas que se les proponen, lo cual en algunos casos, se generaliza a la actividad física en general. Enfatizamos que cualquier conocimiento, disciplina o actividad sólo resulta verdaderamente efectivo cuando logramos que adquiera un sentido real para el alumno.

La educación física, como la artística, la laboral u otros tipos e actividades del curriculum orientadas al desarrollo de aspectos concretos de la persona, son importantes tambiém por la implicación de nuevos espacios sociales para su realización, favoreciendo, así, la adopción de nuevos papeles y el establecimiento de nuevas amistades que pocas veces son explotados en el desarrollo de estas actividades. 
La Educación Artística, aunque parezca distante de lo que tradicionalmente ha sido entendido como salud, puede jugar un importante papel en la promoción de ésta. Bien diseiíada, es una disciplina que, ante todo, constituye un espacio para la expresión creativa individual. En él el alumno puede expresar, con libertad, formas de percibir el mundo, emociones, intereses específicos e incluso, habilidades que pueden ser importantes en su ralización individual y en la constitución de una auto-estima positiva. También las múltiples formas de expresión artística pueden constituir formas sanas y enriquecedoras de utilización del tiempo libre, las que pueden ser integradas a un modo de vida saludable.

Lamentablemente, como en el caso de la Educación Física, muchas veces no logra cumplir su objetivo, queda como una materia mas del curriculum sin un impacto real en la subjetividad individual de los alumnos en formación.

Hasta aquí hemos hecho referencia a diversas direcciones de la participación de la escuela en la educación y promoción de la salud: brindando cultura general, preparación para el trabajo, conocimientos y hábitos específicos, etc. Ahora vamos a referimos a una dirección que consideramos fundamentaly en relación con la cual se va a expresar la efectividad de las anteriores: la acción educativa de la escuela en la formación de los recursos psicológicos necesarios para un funcionamiento integral de la persona que disminuya su vuInerabilidad a la enfermedad y favorezca la asumción de un modo de vida que pueda considerarse suladable.

La educación ha sido concebida tradicionalmente en su sentido mas estrecho, como enseñanza, y la escuela ha sido diseñada como un espacio de transmición de conocimientos y de desarrollo de hábitos y habilidades específicas. EI papel de la escuela en la formación de la personalidad, lamentablemente ha sido tratado de forma muy insuficiente.

Cuando nos referimos a la personalidad la estamos concibiendo como "una configuración sistémica de los principales contenidos y operaciones que caracterizan las principales funciones del sujeto" (González y Mitjáns, 1989:16). La personalidad, así entendida, es un complejo sistema de sentidos donde se integran recursos psicológicos de diferente ordeno Ese sistema constituye el conjunto de recursos subjetivos con los que el sujeto opera en la determinación de sus comportamientos en las múltiples y diversas situaciones en que se encuentra.

La personalidad tiene una determinación compleja, en la cuallo social (la interacción con los otras, la cultura etc.) constituye un elemento esencial. El nifio no nace seguro o inseguro, flexible o rígido, motivado hacia un tipo de actividad o hacia otra, dependi ente o independiente. Es en la compleja evolución de su historia individual donde se va constituyendo la configuración de recursos psicológicos que denominamos personalidad.

Estos recursos se van conformando en función de las características de los múltiples sistemas actividades-comunicación en los que el individuo interactúa a lo largo de su desarrollo. La sociedad como macrosistema y Ia familia y la escuela como sus instituciones básicas, constituyen importantes sistemas actividades-comunicación que participan activamente en el desarrollo de la personalidad.

La escuela constituye un importante espacio comunicativo, es a través de la comunicación que cobran sentidos diferenciados las actividades que se realizan y los conocimientos que se imparten.

Lamentablemente la escuela no está disefiada de forma que la comunicación constituya el eje central de atención y muchas veces el énfasis en la transmición de conocimientos y en el desarrollo de habilidades que la caracteriza, lejos de contribuir a desarrollar recursos subjetivos favorables, estimula la inseguridad, el temor al error, la subvaloración, la desmotivación etc.

Se ha comprobado que hay un conjunto de recursos subjetivos asociados a una personalidad sana y que la escuela puede contribuir a desarrollar, por ejemplo: "riqueza de intereses, capacidad de autodeterminación, flexibilidad para encontrar alternativas adecuadas ante situaciones tensionantes y contradictorias, riqueza de objetivos y planes futuros sobre los cuales el sujeto pueda apoyarse para enfrentar exigencias inmediatas" (González y Mitjáns, 1989:254).

Estos y otros elementos, como capacidad de reflexión personalizada, capacidad para 
estructurar el campo de acción, motivaciones específicas hacia la salud etc, posibilitan también que el sujeto asuma un modo de vida sano, cuyo contenido estará siempre en relación con las posibilidades y límites de la situación social en que vive.

La educación de la personalidad también juega un importante papel en el desarrollo de la creatividad, ya que hemos comprobado que ésta está determinada, entre otros factores, por configuraciones específicas de recursos personológicos. En este sentido afirmabamos:

"La creatividad es expresión de configuraciones personológicas específicas que constituyen variadas formas de manifestación sistémica y dinámica de los elementos estructurales y funcionales de la personalidad que intervienen en el comportamiento creativo: las configuraciones creativas" (Mitjáns, 1995:56).

Entre los recursos personológicos que integran las configuraciones creativas de los sujetos creativos que hemos estudiado, se destacan formaciones motivacionales proyectadas en una dimensión temporal futura, capacidades cognitivas diversas, apertura a la experiencia, flexibilidad, autoconfianza, autodeterminación y cuestionamiento y reflexión personalizados. Puede observarse que muchas de estas características coinciden con las que se consideran propias de una personalidad sana.

Correspondió a la Psicología Humanista norte americana el haber acentuado, en su momento, la interrelación entre creatividad y salud (Maslow, 1979, 1982; May, 1982; Rogers, 1990); interrelación que, actualmente, es reconocida por muy diversos autores.

Através de su producción creativa el sujeto satisface determinadas necesidades y motivos de su personalidad, encuentra posibilidades de expresión propia, experimenta importantes vivencias emocionales e incrementa la confianza en sus posibilidades y realizaciones lo que influye en el desarrollo de su identidad personal. Es por ello que la estimulación de la creatividad y de los recursos presonológicos que están en su base se toma una importante vía de promoción de salud.

Por todas las razones anteriormente esbozadas, contribuir a educar una personalidad rica y sana debe ser un objetivo intencional de la escuela, ya que son precisamente los recursos subjetivos que la integran los que van a participar en la regulación del comportamiento del sujeto en todas las esferas de la vida.

Ella no sólo constituye el núcleo en relación al cual van a adquirir sentido los conocimientos y en general la cultura que la escuela intenta transmitir sino que constituye el sistema regulador del comportamiento del sujeto y en esa codición deviene en mediatizador esencial de la salud.

La institución escolar hoy, enfrenta el desafio de realizar todas las modificaciones que son necesarias en su funcionamiento y disefio par ocupar el lugar esencial que le corresponde en la promoción de la salud humana. Muchas experiencias pedagógicas parciales exitosas indican la posibilidad de estructurar, en contextos escolares específicos, estrategias globales y sistémicas con ese objetivo. Corresponde a profesores, psicólogos escolares pedagogos, orientadores y directivos trabajar mancomunadamente para lograrlo.

\section{Referências}

Bohoslavsky, R. (1987). Orientação vocacional: a estratégia clínica. São Paulo: Martins Fontes.

González, F. (1993). Personalidad, salud y modo de vida. Caracas: Fonde Editorial de la Faculdad de Humanidades, Universidad Central de Venezuela.

González, F. \& Mitjánz, A. (1989). La personalidad: su educación y desarrollo. La Habana: Pueblo y Educación.

Maslow, A. (1979). El hombre autorrealizado. Barcelona: Kairós

Maslow, A. (1982). La amplitud potencial de la naturaleza humana. México: Trillas.

May, R. (1982). A coragem de criar. Rio de Janeiro: Nova Fronteira.

Mitjáns, A. (1995). Creatividad, personalidad y educación. La Habana: Pueblo y Educación.

Poltrony, J. (1989). Modo de vida. Tese de doutorado, La Habana: Universidad de la Habana.

Rogers, C. (1990). El proceso de convertirse en persona. México: Paidós. 